pour celles de l'industrie et de l'art, on a utilisé des figurines encore plus représenta. tives.

L'impression générale qui se dégage de l'Atlas de Salzbourg est qu'il offre, dans un minimum de place, un maximum de matière. On peut donc lui souhaiter, d'être employé au pays comme à l'étranger, par un nombreux public - et aussi d'être suivi par des publications semblables.

\title{
DER SALZBURG-ATLAS
}

Der Aufsatz versucht eine knappe Würdigung des Salzburg-Atlasses zu bieten, der kürzlich als erster österreichischer Landesatlas seit dem Kriege im Verlag Otto Müller in Salzburg erschien und auf 66 Kartenblättern das gleichnamige Bundesland unseres Nachbarstaates zur Darstellung bringt. Als Herausgeber amtete Dozent Dr. Egon Lende, dem ein Stab mit dem dargestellten Objekt vertrauter Spezialisten zur Seite stand. Der Atlas gliedert sich in einen Karten- und Textteil, welch letzterer einer knappen systematischen Landeskunde gleichkommt. Dispositionell bauen beide die Hauptkapitel: Landesnatur, Mensch, Wirtschaft, Geschichte, Kunstgeschichte, Volkskunde und Kultur, Verwaltung und Wohlfahrt auf, worin das Streben der Atlas-Bearbeiter zum Ausdruck kommt, verschiedensten Kreisen und $Z$ wecken zu dienen. Im Rahmen dieser Hauptkapitel breitet der Atlas einen großen Reichtum an Tatsachen aus, der zusammen mit dem Text ein abgerundetes Bild des Landes Salzburg entwirft. Was die Graphik anbetrifft, darf sich das Werk mit besten Mustern messen. Wenn auch eine Reihe von Karten zu Kartogrammen geformt sind, tat dies der Exaktheit keinerlei Abbruch. Im Gegenteil gewann dadurch das Werk an Klarheit und Lesbarkeit, was insbesondere im Blick auf seine vielfältigen $Z_{w e c k e}$ wichtig ist. Die Karten haben die Maßstäbe 1:500 o00 und 1:1000 000 , worin sich ihre Möglichkeiten manifestieren. Sie sind nach Farbwahl und Signaturen ansprechende und einprägsame Bilder. Als Gesamteindruck läßt sich deshalb vom Salzburg-Atlas sagen, daß er bei Beschränkung auf knappsten Raum ein Maximum an Form und Inhalt bietet. Man kann ihm deshalb im In- und Ausland nur zahlreiche Benützer - und Nachfolger - wünschen.

\section{ZUR KENNTNIS DER VEGETATIONSSTUFEN IM MITTLEREN OSTEN}

\section{Constantin Regel}

\section{EINFÜHRUNG}

Bei der Fahrt von Baghdad nach Jerusalem überschreitet man hinter Amman das Tal des Jordan, dessen Vegetation sich von der umliegenden Gegend stark unterscheidet und die sich nicht in die Folge der Vegetationsstufen einfügen läßt, die wir im Mittleren Orient beobachten können.

Es unterliegt keinem $Z$ weifel, daß sich die Vegetationszonen der Ebene mehr oder weniger in den Vegetationsstufen der Gebirge widerspiegeln, wobei aber die zu beobachtenden Unterschiede in den verschiedenen Gebirgen als eine Folge der lokalen Verhältnisse und der Geschichte gedeutet werden müssen.

Die Analogie der Vegetation der Zonen und der Stufen bedingt die von mir 1939 formulierte Gesetzmäßigkeit, daß der Klimaxverein an der oberen Waldgrenze eines Gebirges der gleiche ist, der auch in der nördlich davon gelegenen Vegetationszone vorherrscht. Der Klimaxverein am Fuße dieser Gebirge bildet weiter im Süden den Klimaxverein an der oberen Waldgrenze.

Wir sehen diese Gesetzmäßigkeit auch in den Ländern des Mittleren Ostens bestätigt. So liegt Iraq 1 in der Landschaftszone der Wüste. Diese zerfällt in zwei Unterzonen, die der eigentlichen und die der Halbwüste, die beide den Iraq durchziehen. Baghdad und Basrah das alte Babylonien - liegen in der Unterzone der eigentlichen Wüste: die Vegetation fehlt ganz oder ist nur sehr spärlich und bedeckt weniger als die Hälfte des grauen bis gelben Wüstenbodens. Ackerbau ist nur bei Berieselung möglich, die Niederschläge sind gering, das

1 Im Iraq ist bei dem Fehlen von Düngung und der Felderwirtschaft die Hälfte des bebauten Bodens Brache. 
Jahresmittel beträgt nur $200 \mathrm{~mm}$ und weniger, die Brache besteht aus Prosopis starkiana (eine kleine Mimosacee) und Alhagi maurorum (Maurischer Kameldorn), die Dattelpalme bildet an den Ufern des Euphrat und Tigris ungeheure Waldungen von Baghdad abwärts bis zum Persischen Meerbusen hin. Der Norden des Iraq, also das alte Assyrien, gehört zur Unterzone der Halbwüste. Das Jahresmittel der Niederschläge beträgt hier mehr als $200 \mathrm{~mm}$, in Mossul z. B. $411 \mathrm{~mm}$ (Boesch 1939).

Die Vegetation bedeckt mehr als die Hälfte des häufig rot gefärbten Bodens, bildet jedoch keinen geschlossenen Teppich. Ackerbau ist auch ohne Berieselung möglich, da die Niederschläge des Winters und Frühjahrs die Reife des Getreides im Frühling ermöglichen. Die Brache besteht aus zahlreichen Kräutern, die Dattelpalme kommt nicht oder nur ganz vereinzelt vor. Dies sind nur einige wenige Merkmale der beiden Unterzonen der Wüste (siehe auch Regel 1956). Im Norden und Nordwesten geht die Landschaftszone der Wüste in die der Trockenzone über, deren westlicher, vom Ozean beeinflußter Teil, das Mittelmeergebiet, der östliche, kontinentale, die Steppe ist.

Im Mittleren Osten läßt sich dieser Übergang z. B. auf der Fahrt von Mossul nach Aleppo gut beobachten. Durch Syrien und die Türkei erstrecken sich zu beiden Seiten der Bahnlinie bei Nisibin-Mardin-Urfa weite Getreidefelder an Stelle der einstigen unberührten Steppe. Hinter Haleb (Aleppo) beginnt das Mediterangebiet kenntlich an den immergrünen Sträuchern und den aus Pinus Brutia (Mittelmeer Kiefer) bestehenden Wäldern.

Auf dem Balkon und in Mitteleuropa grenzen Steppe und Mediterrangebiet an die Zone der Laubwälder. Diesen Zonen begegnen wir auch in den Gebirgen des Iraq als Vegetationsstufen.

Im Nordosten von Mossul, also in der Unterzone der Halbwüste, erheben sich die Gebirge Kurdistans bis zu 3500 m absoluter Höhe. Oberhalb der Halbwüste oder vielmehr der an ihrer Stelle entstandenen Brache beginnt die Steppe und als Relikt eines einst feuchteren Klimas die Mediterranvegetation ${ }^{2}$. In ca. 600-700 m treten die ersten Eichen als Überreste des zerstörten Eichenwaldes auf, der weiter oben alle Hänge bedeckt, bis ca. 1800-2000 m reicht und die alpine Waldgrenze bildet. Es bestehen hier also zwei Waldgrenzen, die untere, an der Grenze zur Steppe und die obere. Die alpine Stufe ist hier infolge der großen Trockenheit im Sommer nicht mit der der Gebirge Mitteleuropas zu vergleichen, erst weiter oben, wie z. B. auf dem $3500 \mathrm{~m}$ hohen Hassar Ost begegnen wir Pflanzen der alpinen Stufe Mitteleuropas (siehe übrigens REgEL 1956).

Die Stufe des Eichenwaldes der Gebirge Kurdistans entspricht der Zone des Laubwaldes in Mitteleuropa und zwar infolge der größeren Trockenheit deren südlichem Teil, die dem Quercus pubescensgürtel (Schmid 1949), entspricht. (Bовек 1951) nennt diese den Zagros-Eichenwald, der zu den Trockenwäldern gehört und in den an den Iraq angrenzenden Teilen der Türkei und des Iran verbreitet ist.

Das Zagros-Gebirge weiter im Süden, also im Osten der irakischen Grenze, liegt schon innerhalb Irans; doch geht hier wie z. B. bei Khanikin, die Wüste in eine Steppe über, die alle Anhöhen bedeckt. Die Waldgrenze wird also, wie in den Gebirgen des türkischen Kurdistans, von den Laubwäldern gebildet, die weiter im Norden, in Europa in der Ebene verbreitet sind, womit sich die eingangs erwähnte Gesetzmäßigkeit bestätigt.

\section{DAS GEBIET ZWISCHEN BAGHDAD UND JERUSALEM}

Die Stufenfolge der Vegetation der irakischen Gebirge entspricht also den Vegetationszonen der Ebene. Wie ist es nun dort, wo die in Jordanien und Israel, die tiefe

2 Eingehendere Studien über die pflanzengeographischen Verhältnisse des Iraq sind in Vorbereitung, doch sind die vom Verfasser gemachten Aufzeichnungen, auch die, die dem vorliegenden Artikel zugrunde liegen, nur unvollständig, da der mit dem University College in Baghdad abgeschlossene Vertrag vor Abschlu $B$ der Arbeiten nicht mehr erneuert wurde.

Eine Arbeit über die Mediterranvegetation des Iraq ist in Vorbereitung. 
Senke des Toten Meeres die normale Verteilung der Stufen stört? Wir wollen diesen Fragen an Hand einer Fahrt von Baghdad nach Jerusalem nachgehen.

Baghdad liegt in $32 \mathrm{~m}$ Meereshöhe in der Unterzone der eigentlichen Wüste. Allerdings ist diese durch die schon seit der Zeit des alten Babylonien begonnenen Berieselung stark verändert und in Getreidefelder umgewandelt worden. Alluvialer Boden mit Brachen aus Prosopis und Alhagi bedeckt, bildet die Grundlage. Hinter Abu Chraib, einer durch Berieselung entstandenen Oase mit dichtem Baumwuchs, beginnt die eigentliche Wüste auf sandig-kiesigem Boden, die sich bis Faluja am Euphrat erstreckt, und die wir nach der charakteristischsten auf ihr vorkommende Pflanze, die Aristida plumosa(Federgras)-Wüste nennen wollen. Dazu kommen hier Poazinaica (Rispengras), Astragalus spinosus (Stacheliger Tragant) und andere Arten hinzu.

Bald hinter Faluja verläßt man das Alluvial, es beginnt die Shamiya, der arabische Block (Boesch 1939), die steil gegen das Alluvial des Euphrat abstürzt. Sie besteht aus sedimentären Gesteinen, die nach MacFaden (siehe Kellersohn 1953) dem Pliozän und Obermiozän und westlich von Ramadi dem Miozän angehören.

Während sich am Fluß Getreidefelder und Palmenhaine ablösen, erstreckt sich oben die Wüste bis nach Jordanien und Syrien hinein. Je nach dem Untergrund lassen sich verschiedene Typen der Wüste unterscheiden, wie die kiesig-sandige Aristida plumosa-Wüste. In den Vertiefungen an der von Ramadi nach Rutba führenden Straße, wo sich nach den Niederschlägen die Feuchtigkeit länger hält, wird die Vegetation reicher, und die Gräser bilden stellenweise einen dichteren Rasen. Hordeum murinum (Mäusegerste), Papaver Rhoeas (Feuermohn), Matricaria Chamomilla (Echte Kamille) u. a. Arten kommen hier vor.

In Jordanien beginnt die Lava-Wüste, die sich kilometerweit auf hügeligem Gelände erstreckt und vegetationslos ist (S. FISHER). Es ist die Syrische Wüste, die nach Zohary (1939) zur Unterzone der echten Wüsten gehört, deren Teilregionen von EIg das Saharo- das Saharo-Indische Territorium genannt werden.

Zerka und Amman gehören bereits zum Irano-Turanischen Territorium, das in der Unterzone der Halbwüste liegt. Obwohl, die Vegetation durch den Menschen stark verändert wurde und stellenweise einen wüstenhaften Charakter angenommen hat, weist sie auf Iranisch-Turanische Zugehörigkeit hin. Der sandig-kiesig-felsige Boden ist mit spärlicherVegetation bedeckt (u. a. Matricaria Chamomilla, Artemisia spec., Papaver Rhoeas u. a.). Nach Erg (1938) liegt Amman an der Grenze zwischen dem Irano-Turanischen und mediterranen Territorium. Der rote Boden, die Ölbäume, Feigen- und Mandelbäume auf den Terrassen, Opuntia, Anschusa spec. [Ochsenzunge] u. a. sind dafür bezeichnend. Nerium Oleander an Bächen weist auf mediterranen respektive auch auf Steppeneinschlag, Papaver Rhoeas auf Halbwüste, laubabwerfende Gehölze auf die Stufe der Laubwälder hin. Doch ist die Gegend stark verwüstet, die ursprüngliche Vegetation vernichtet worden und hat stellenweise direkt sekundären Felswüsten und Karst Platz gemacht. Anschließend kommt man wieder in das Iranisch-Turanische Territorium, das nach einer Unterbrechung im Jordantal einen schmalen Streifen an den Hängen bedeckt, um schließlich bei Jerusalem ins Mediterrangebiet überzugehen (siehe Karte bei EIG 1.c.).

Vergleichen wir mit den Kurdischen Gebirgen, so fehlt hier eigentlich die Stufe des Trockenwaldes mit laubabwerfenden Bäumen. Die Erhebungen sind zu wenig hoch, um einen solchen $\mathrm{zu}$ ermöglichen. Immerhin finden sich einzelne Elemente an den Hängen, und auch im Israelischen Gebiet sind stellenweise größere Bestände von laubabwerfenden Eichen vorhanden.

Das Jordantal bildet eine Unterbrechung in der Verteilung der Stufen; denn es liegt, soweit es nicht in Kulturland umgewandelt erscheint, in der Wüste, die an beiden Ufern des Toten Meeres überaus charakteristisch ausgeprägt ist (siehe ZoHARY und Orshansky). Tamariceten (Tamarisken-Bestände) am Ufer des Flusses erinnern an die Alluvialvegetation in der Wüste des Iraqs, die Palmenwälder an die Euphrat 
und Tigris. Höher, wie auch weiter im Westen Israels fehlen diese Palmenwälder. Darauf weist schon De Candolle (1896) hin, der die fruchttragenden Palmen im Tale des Jordan erwähnt, im Gegensatz zu den keine Früchte tragenden im übrigen Palästina. Daraus können wir schließen, daß das Tal des Jordan dem Gedeihen der Dattelpalmen förderlicher ist, als die weiter westlich gelegenen Gegenden und in klimatischer Hinsicht dem Klima der echten Wüste mit ihren Palmenwäldern, entspricht.

Das Tal des Jordan liegt ca. $400 \mathrm{~m}$ unter dem Meeresspiegel. Am Meeresniveau erstreckt sich die Trockenzone (Mediterrangebiet und Steppe), wie aus der Karte von Bоyко (1954) ersichtlich ist. Nach Osten hin müßte diese Trockenzone in der Nähe von Amman allmählich in die Wüste übergehen, wie wir es auch im Nordwesten von Aleppo gegen Mossul hin sehen, oder beim Übergang von der Ebene zu den Kurdischen Gebirgen und zum Zagros. In Wirklichkeit wird sie von der in der Senke des Jordan und des Toten Meeres liegenden Wüste unterbrochen.

Auf dem Profil bei Kümmel (1949) finden wir diese Abweichung in der Vegetation als «tropische Enklaven» eingezeichnet. Die Verfasserin stützt sich auf die Untersuchungen von EIG :

«Im tiefen Tal des Hebron, lesen wir, wachsen im Gegensatz zur Wüste entlang des Flusses Auenwälder mit Populus euphratica (Euphratpappel) und Salix babylonica (BabylonWeide). Die Wüstenregion zeigt jedoch am Strande des Toten Meeres wildwachsende Dattelpalmen (Phoenix dactylifera) an, auch Acacia Arten (Acacia seyal) und Zizyphus spina Christi (syrischer Christdorn), in feuchteren Stellen Elaeagnus angustifolia (Ölweide). Besonders auffallend und anziehend sind die Reliktinseln sudanischer Savannenflora Afrikas, die am Süd- und Ostufer des Toten Meeres als «Sudanische-Dekkanische Enklave» EIGs vorkommen und eine ganz eigenartige, üppige, fremdartig anmutende Flora besitzen. Besonders zu nennen sind: Zizyphus spina Christi, Moringa aperta, Capparis decidua (Kapernstrauch), Acacia seyal, die inmitten der Wüstenlandschaft des Toten Meeres im Oktober in voller Blütenpracht erscheinen. Bezeichnend sind auch noch Acacia tortilis und Panicum turgidum.»

Diese Feststellung stimmt mit unsern Beobachtungen überein, die zeigen, da $\beta$ unterhalb des Meeresspiegels die Vegetationsstufe der Wüste auftritt. Auch auf der Karte von EIG liegt das Tal des Jordan und des Toten Meeres inmitten des Saharoindischen Territoriums, $d . h$. in der Unterzone der echten Wüste.

\section{ZUSAMMENFASSUNG}

Wir können aus dem hier Gesagten folgenden Schluß ziehen, der die oben formulierte Gesetzmäßigkeit ergänzt:

Die Vegetationsstufen oberhalb des Meeresniveaus wiederholen sich in den weiter im Norden liegenden Vegetationszonen, die Vegetationsstufen unterhalb des Meeresniveaus wiederholen sich in den weiter im Süden liegenden Vegetationszonen. Da jedoch im Süden der Trockenzone (Mediterrangebiet und Steppe) die Wüstenzone beginnt, und in Palästina und im westlichen Jordanien die Trockenzone am Meeresniveau liegt, so befindet sich unter dem Niveau des Meeres, also im Tal des Jordan und am Toten Meere ebenfalls die Wüste. Doch sind auf unserer Erde zu wenig genügend tiefe Depressionen vorhanden, als daß diese Gesetzmäßigkeit durch weitere Beispiele bestätigt werden könnte. Doch müßte in noch größerer Tiefe die echte Tropenzone auftreten.

Einen ähnlichen Gedanken finden wir in dem vom bekannten Geologen OвRuтSCHEW (1937) verfaßten phantastischen Roman «Plutonia» wieder: die Erde ist hohl und beim Eindringen in diesen Hohlraum von der Arktis aus, gerät man sukzessive durch verschiedene Vegetationsstufen bis in die tropische Vegetation hinein.

LITERATUR: BoвEk, H.: Die natürlichen Wälder und Gehölzfluren Irans. Bonner Geogr. Abh., Heft 8, 1951. - BoEsch, H.: El-Iraq. Economic Geography 15, Worcester, 1939. - BоYко, H. : New Plant-Geographical Subdivision of Israel. Vegetation V-VI, Den Haag, 1954. - De CANDOLLE, A.: L'Origine des plantes cultivées. Paris, 1896. - EIG, A.: On the phytogeographical subdivision of Palestine. Palest. Jour. of Botany J. Series I-1. Jerusalen, 1938. - FisheR, W. B.: The 
Middle East, a physical, social and reginal Geography. London, 1952. - Kellersohn, H.: Die Landwirtschaft im Irak. Erdkunde VIII, Lfg. 4, Bonn 1953. - KüMmEL, K. : Die Stellung Südfrankreichs und der Krim im West- und ostmediterranen Vegetationsprofil. Bonn, 1949. - MouTERdE, P.: La Flore Jebel. Druze, 1953. - Obrutschew, W. A.: Plutonia. Moskau-Leningrad, 1937 (Russisch). - Regel, C.: Geobotanische Beobachtungen auf einer Reise in Marokko und der Sahara. Ergebn. Internat. Pflanzengeogr. Exkursion durch Marokko und Westalgerien 1936. Veröffentl. Geobot. Institut Rübel 14. Bern, 1939. - Regel, C.: Irak und Spanien. Veröffentl. Geobot. Institut Rübel. Ergebn. Intern. Pflanzengeogr. Exkursion Spanien. Bern, 1956. - Schmid, E.: Prinzipien der natürlichen Gliederung der Vegetation des Mittelmeergebietes. Ber. Schweiz. Bot. Gesellsch. 59, Bern, 1948. - Zohary, M.: The knowledge of the flora of the Syrian desert. Pal. Journ. of Botany J. Series I, Nr. 3. Jerusalem, 1939. - Zohary, M. and ORshansky, A.: Structure and Ecology of the Vegetation of the Dead Sea. Region of Palestine. Pal. Journ. of Botany J. Series IV Nr. 4, 1949.

\title{
CONCERNING THE BELTS OF VEGETATION IN THE MIDDLE EAST
}

The valley of the Jordan between Amman and Jerusalem has a quite peculiar vegetation which does not correspond to the series of vegetation-zones in the Middle East. The belts of vegetation found between the foot to the summit of the mountains, e. g. in the Mediterranean region and in Iraq, correspond more or less to the zones distinguishable in the plain from the South to the North. In the vallay of the Jordan, situated below the level of the Mediterranean Sea, the belts correspond to the zones lying not to the North (Mediterranean region), but to the South (Desertzone). The desert vegetation on the shores of the Jordan and the Red Sea is an isolated ocurrence in the Mediterranean region.

\section{CHICAGO ALS HAFENSTADT}

\section{Hans Boesch}

\author{
Mit 3 Figuren
}

Die Rolle, welche Chicago als Hafenstadt spielt, wird neben allen anderen Funktionen dieser Metropole bei uns häufig übersehen. Die Erstellung des Großschiffahrtsweges von den Großen Seen zum Atlantischen Ozean dürfte in den kommenden Jahren die Bedeutung des Hafens von Chicago noch vergrößern und zu wesentlichen strukturellen Veränderungen des amerikanischen Wirtschaftsraumes führen. Als Unterlagen für die folgende Darstellung dienten neben den amtlichen Statistiken in erster Linie verschiedene in «Economic Geography» erschienene Artikel von H. MAYER, Professor an der University of Chicago, und an Ort und Stelle gesammelte Informationen.

Die Siedlung Chicago wurde an einer der zahlreichen niedrigen Wasserscheiden, einer sogenannten Portage, angelegt, über welche das Gebiet der Großen Seen und des St. Lawrence Rivers mit dem Mississippi in Verbindung steht. Sowohl während der Exploration wie auch in den Anfängen der Siedlungsnahme und wirtschaftlichen Erschließung spielten im Innern der Vereinigten Staaten die Wasserwege die Hauptrolle als Verkehrsträger. Vor dem Bau des Erie-Kanales von Buffalo zum Hudson und nach New York (1825) bildeten der Mississippi und seine Zuflüsse die wichtigsten Verkehrslinien: Dem Ohio folgte der Hauptvorstoß der Siedler, St. Louis wurde zum natürlich gegebenen Mittelpunkt, nach dem Golf von Mexico fand der Handel seinen Ausgang. Mit dem Bau des Erie-Kanals begann der Mississippi «aufwärts zu fließen», New York gewann seine dominierende Stellung an der Atlantikküste und Chicago, der am weitesten westlich und südlich gelegene Punkt an den Küsten der Großen Seen, begann seinen großartigen Aufstieg. 1830 war Chicago noch eine kleine Grenzsiedlung; 1833 wurde es Town, 1837 City und 1890 stieg es zur zweitgrößten Stadt der Vereinigten Staaten empor. Der Seeverkehr spielte vor allem in den Anfängen eine entscheidende Rolle für die Entwicklung der Stadt. Für die Massengüter, Getreide und Holz, welche über Chicago verschifft wurden, blieb diese Bedeutung auch bestehen, als 1852 die erste Eisenbahn Chicago erreichte. 